\title{
Erratum to: Large-scale image colorization based on divide-and-conquer support vector machines
}

\author{
Xinyu $\mathrm{He}^{1} \cdot$ Bo-Wei Chen ${ }^{1}$ - Wen $\mathrm{Ji}^{2}$. \\ Seungmin Rho $^{3}$. Sun-Yuan Kung ${ }^{1}$
}

Published online: 18 March 2016

(C) Springer Science+Business Media New York 2016

\section{Erratum to: J Supercomput DOI 10.1007/s11227-015-1414-z}

The authors would like to correct the authorship credits of the original publication. Xinyu He owned the intellectual property of the majority of the methodologies and source codes in the original paper. The corrected authorship list is given in this erratum.

The online version of the original article can be found under doi:10.1007/s11227-015-1414-z.

$\bigotimes$ Xinyu $\mathrm{He}$

xinyuhe@princeton.edu

1 Department of Electrical Engineering, Princeton University, Princeton, NJ, USA

2 Institute of Computing Technology, Chinese Academy of Sciences, Beijing, China

3 Department of Multimedia, Sungkyul University, Anyang, South Korea 